\title{
Wind Speed Inversion in High Frequency Radar Based on Neural Network
}

\author{
Yuming Zeng, ${ }^{1}$ Hao Zhou, ${ }^{1}$ Hugh Roarty, ${ }^{2}$ and Biyang Wen ${ }^{1}$ \\ ${ }^{1}$ School of Electronic Information, Wuhan University, Wuhan 430072, China \\ ${ }^{2}$ Institute of Marine and Coastal Sciences, Rutgers University, New Brunswick, NJ 08901, USA
}

Correspondence should be addressed to Hao Zhou; zhou.h@whu.edu.cn

Received 6 April 2016; Accepted 14 August 2016

Academic Editor: Khalid El-Darymli

Copyright (C) 2016 Yuming Zeng et al. This is an open access article distributed under the Creative Commons Attribution License, which permits unrestricted use, distribution, and reproduction in any medium, provided the original work is properly cited.

Wind speed is an important sea surface dynamic parameter which influences a wide variety of oceanic applications. Wave height and wind direction can be extracted from high frequency radar echo spectra with a relatively high accuracy, while the estimation of wind speed is still a challenge. This paper describes an artificial neural network based method to estimate the wind speed in HF radar which can be trained to store the specific but unknown wind-wave relationship by the historical buoy data sets. The method is validated by one-month-long data of SeaSonde radar, the correlation coefficient between the radar estimates and the buoy records is 0.68 , and the root mean square error is $1.7 \mathrm{~m} / \mathrm{s}$. This method also performs well in a rather wide range of time and space ( 2 years around and $360 \mathrm{~km}$ away). This result shows that the ANN is an efficient tool to help make the wind speed an operational product of the HF radar.

\section{Introduction}

After Crombie explained the distinct characteristic of the sea echo Doppler spectra obtained by high frequency (HF) radar with Bragg scattering effect, the potential of the HF radar in remote sensing of the sea state parameters was recognized [1]. Barrick further derived the well-known firstand second-order radar cross section (RCS) equations and directly pushed forward the applications of the HF radars $[2,3]$. Due to the unique capabilities of large-area, real-time, and all-weather monitoring over the sea surface, HF radar now has gained much attention and has been widely installed and operated all over the world.

As one data product of HF radar, wind speed estimate over the sea is of great help to the development and utilization of the sea resources. It is important for a wide variety of coastal and marine activities, such as sailing, fishing, and wind power generation. Wind over the oceans is typically strong and steady, representing a rich source of renewable energy [4]. It also plays a key role in many oceanographic processes including the air-sea interaction and the climate systems in both regional and global scales. After thirty years of development, the extraction of current velocity and wind direction has achieved great success and they have become mature products of the HF radar. The wave height estimation has also reached relatively high accuracy. However, the wind speed estimation is still a challenge. The conventional wind speed estimation is via an indirect process; that is, it is not directly extracted from the radar echo spectrum but from the wave height and period estimates. It is the sea wave that generates the radar echo. The solutions to the wind-wave relationship are mainly based on empirical or semiempirical models, which may have different performances in different sea area. The errors in both of the wind-wave model and the wave estimation make it much more difficult to extract the wind speed.

The relationship between the wave parameters and wind speed has been studied for several decades. The common wave parameters used to estimate the wind speed are significant wave height $H_{s}$ and dominate wave frequency $f_{m}$. The SMB relationship is such a semiempirical model [5-9]. It has been widely applied in the marine forecasting. However, the wind speed cannot be given in an analytical solution but should be sought by iteration, which depends on a proper initial value and may converge to a wrong estimate. Power regression is another method reported to estimate the wind speed, but it may lead to big errors for both low and high 
TABLE 1: Performance comparison of different algorithms.

\begin{tabular}{|c|c|c|c|c|c|c|}
\hline & \multicolumn{6}{|c|}{ Algorithm } \\
\hline & Linear regression & Nonlinear regression & $\mathrm{RBF}$ & GRNN & ANFIS & $\mathrm{BP}$ \\
\hline RMSE (m/s) & 2.50 & 2.65 & 2.72 & 2.69 & 1.61 & 1.66 \\
\hline $\operatorname{MAE}(\mathrm{m} / \mathrm{s})$ & 2.00 & 2.05 & 2.11 & 2.09 & 1.22 & 1.3 \\
\hline $\mathrm{CC}$ & 0.67 & 0.62 & 0.70 & 0.83 & 0.87 & 0.89 \\
\hline SI & 0.38 & 0.41 & 0.42 & 0.41 & 0.25 & 0.25 \\
\hline
\end{tabular}

wind speeds due to the high simplification of the wind-wave relationship [10]. Other indices have also been reported to contain wind speed information, for example, the widening of the first-order Bragg peaks and the frequency position of the second-order peak; however, these methods show a limited applicability, and the estimation error does not show an obvious decrease [11, 12]. To achieve better knowledge of the wind-wave relationship, Mathew and Deo introduced the artificial neural network (ANN) into the wind speed extraction [13]. The ANN is a totally data-driven, empirical way that can inverse the nonlinear relationship between the wind speed and other radar-extracted sea state parameters. Four parameters, that is, the significant wave height, average wave period, wave direction, and wind direction, are input to the ANN, and the output gives the corresponding wind speed estimate. The training process relies on the buoy measurements. The results show that the wind speed estimation error has reached an acceptable level and the ANN is a powerful tool for wind speed inversion. Not alone, the ANN method is also used to inverse the wind speed directly from the firstorder Bragg peaks in HF radars [14]. Although this firstorder method has a narrower range of wind speed estimation because the Bragg wave involved is easier to be blown into saturation under a wind speed not very high, the result is also encouraging and once more shows the capability of the ANN.

In this paper, we continue to use the ANN method proposed by Mathew and Deo for the wind speed extraction in HF radar. Differently, with the consideration that the wave direction estimate is often not very accurate, especially in the small-aperture radars such as the SeaSonde to be discussed here, we remove it from the input. That is, the ANN involved in this paper is a three-in-one-out network. The historical data recorded by the National Data Buoy Center (NDBC) buoy number 44025 near the east coast of the USA are used to train this ANN, and then the wave height, period, and wind direction estimates achieved by the HF radar are input to it to give the wind speed estimate. To cover different sea states, data of a whole year are used in the training process. As a result, the ANN is found to be applicable in rather wide ranges of both time and space. On one hand, the estimation performance of the trained ANN is nearly independent of the data time; for example, the ANN trained by the buoy data in 2008 still works well on the data in 2013. On the other hand, the trained ANN also works well on the data from other buoys as far as several hundreds of kilometers away. The radar involved is a $13 \mathrm{MHz}$ SeaSonde system located at the BRMR site in New Jersey, USA, which serves as part of the Mid Atlantic HF radar network. The radar wind speeds in May, 2012, are estimated by the ANN, which shows a relatively high correlation coefficient of 0.68 and a small root mean square error (RMSE) of $1.7 \mathrm{~m} / \mathrm{s}$ compared with the buoy measurements. These results are much better than those obtained by the SMB method and the power regression method. The ANN method opens the possibility of making the wind speed estimate an operational data product of the HF radar.

\section{Methodology}

The ANN is a statistical learning algorithm inspired by animal brains which can perform fast parallel, nonlinear computing and complicated information processing. It can be used to estimate unknown functions that depend on a number of inputs. The ANN consists of a group of interconnected nodes (neurons) and their mutual connections [15]. Each node means a specific transfer function, and different weights are assigned to the interconnections between the nodes, which store the memories of learning. The ANN learning is just accomplished by adjusting the weights of the interconnections based on a given training data set. The backpropagation (BP) neural network is an algorithm developed to train a multilayer ANN so that it can learn the appropriate internal representations to allow it to learn arbitrary input-to-output mappings [16]. The BP calculates the gradient of the network error with respect to the weights and then updates the weights opposite to the gradient direction. The BP ANN has been the most widely applied network in classification, data compression, functional approximation, and nonlinear regression due to its strong abilities of nonlinear mapping and generalization.

To confirm that the BP ANN is the best choice for wind speed inversion, we evaluated six different algorithms, as displayed in Table 1. In this evaluation, the buoy data in 2011 were used to get the best parameters of different algorithms, while the buoy data during 2012 were used to test the different algorithms. For all six algorithms, we found that the BP had the best fitting ability. The linear and nonlinear statistical regressions lack sufficient capacity to fit. It can only fit the wind-derived waves for significant wave height as single input parameter. The fitting abilities of RBF (Radial Basis Function) and GRNN (Generalized Regression Neural Network) in wind speed inversion are not as well as BP ANN and ANFIS. As a combination of fuzzy logic and neural network, ANFIS (Adaptive Neural Network Based Fuzzy Inference System) has good performance but its correlation coefficient is slightly worse than that of BP.

In this study, we construct a three-layer BP network to map the relationship between the wind speed and three other sea state parameters, say the significant wave height, average 


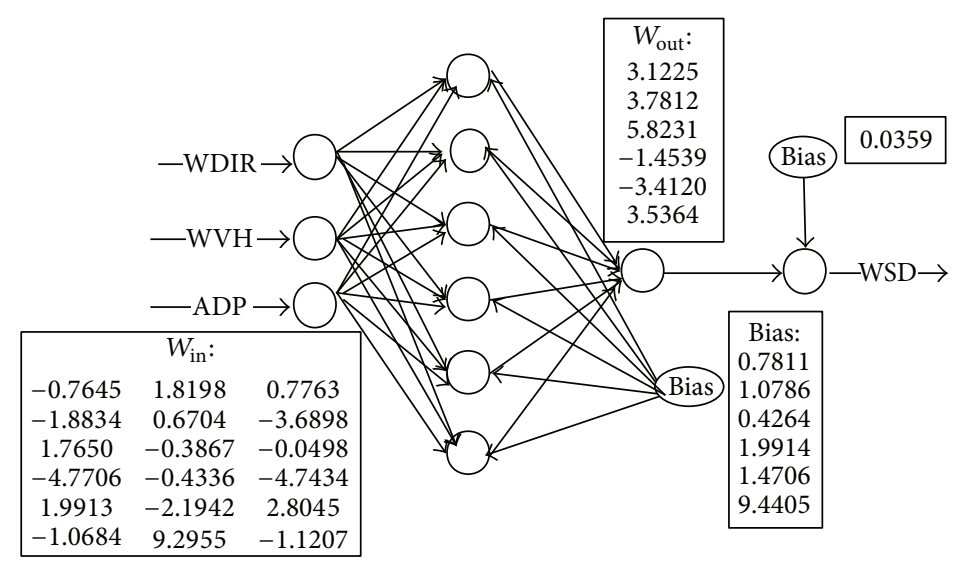

FIgURE 1: The structure of the ANN.

wave period, and wind direction. The hidden layer has six neurons, which is found to offer satisfactory estimation performance. More neurons will make the training slower, while fewer neurons may lead to an insufficient mapping ability. The transfer function of the hidden layer is the logsigmoid function, and that of the output layer is the tansigmoid function. The log-sigmoid function can compress the output value into the range of 0 to 1 , and the tan-sigmoid function can compress the output value into the range of -1 to 1 . The sigmoid function is advantageous because it can accommodate large signals while allowing pass of small signals without excessive attenuation. The structure of the ANN is shown in Figure 1. To improve the generalization ability of the ANN and avoid overtraining, we use the Bayesian regularization training method, which can improve the performance of the small-scale network by adding the mean sum of the squares of network weights to the objective function [17, 18]. After the ANN converges, it is able to output the wind speed estimate from the input of HF radar measurements.

Each training data set covers a whole year of data from the NDBC buoy number 44025 , located at $40^{\circ} 15^{\prime} 3^{\prime \prime} \mathrm{N}, 73^{\circ} 9^{\prime} 52^{\prime \prime} \mathrm{W}$, with the wind speed, significant wave height, average wave period, and wind direction being recorded every hour. The ANN trained by the buoy data in 2011 is then applied on the data achieved by the $13 \mathrm{MHz}$ SeaSonde radar which is located at the BRMR site $\left(39^{\circ} 24^{\prime} 30^{\prime \prime} \mathrm{N}, 74^{\circ} 21^{\prime} 41^{\prime \prime} \mathrm{W}\right)$ near New Jersey, USA, in 2012. The geographic map is shown in Figure 2 for clarity. The total number of pieces of the buoy data in 2011 is 6292.

To further evaluate the performance of the ANN method, the SMB method and the power regression method are also used on the radar measurements to estimate the wind speed. The SMB relationship is given by

$$
\frac{g H_{s}}{V_{10}^{2}}=0.26 \tanh \left[\frac{\left(f_{m} V_{10}\right)^{-3 / 2}(3.5 g)^{3 / 2}}{100}\right],
$$

where $g$ is the gravity acceleration, $H_{s}$ is the significant wave height, $f_{m}$ is the peak frequency of the wave height spectrum, and $V_{10}$ is the wind speed at a height of $10 \mathrm{~m}$ above the sea

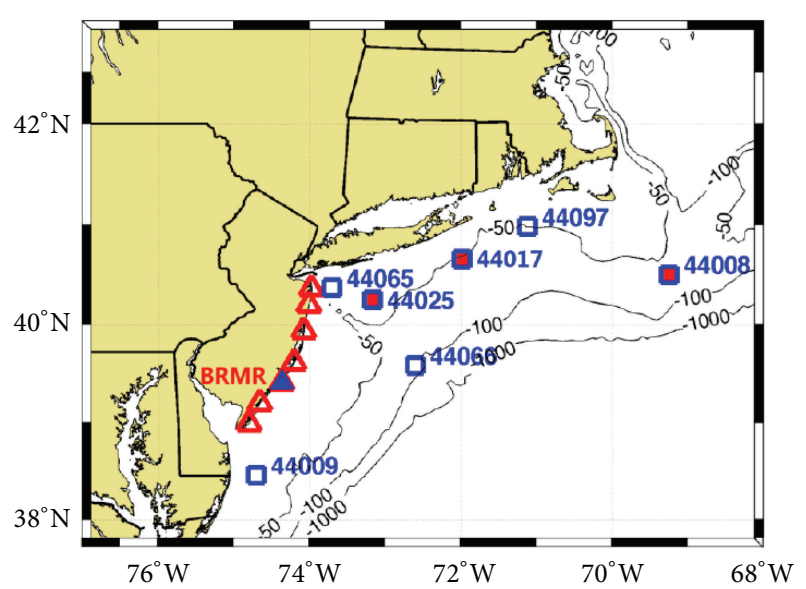

Figure 2: Geographic map of the buoys and the SeaSonde site involved (reproduced from [19]).

surface. There is not an analytical solution for $V_{10}$, so it should be approached by iteration [6-8]. This equation stands for fetch restricted conditions [9]. The power regression method further ignores the impact of the wave period and directly estimates the wind speed from the wave height by use of the power model:

$$
V_{10}=\alpha H_{s}^{\beta},
$$

where $\alpha$ and $\beta$ are two constant coefficients which can be determined by fitting to the buoy data.

\section{Data Processing Results}

The buoy data set of a whole year is used to train the ANN. We aim to extract wind speed from the HF radar data in 2012, so we choose the buoy data in 2011 to train the ANN. The average wave heights and wind speeds recorded by the NDBC buoy number 44025 in each month of 2011 and 2012 are listed in Tables 2 and 3, respectively. We can see that the average sea states are lower during the summer months, say May till August, than the winter months, say November till February. 


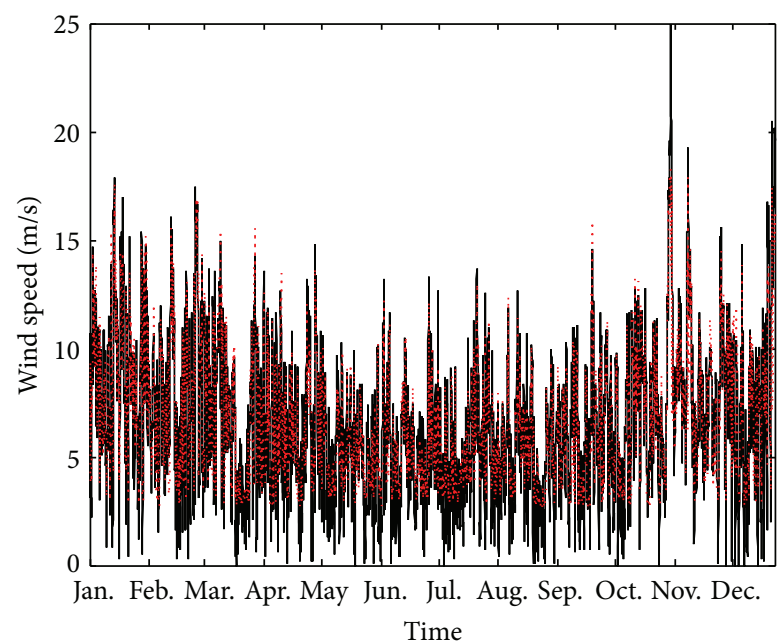

- Buoy

ANN

(a)

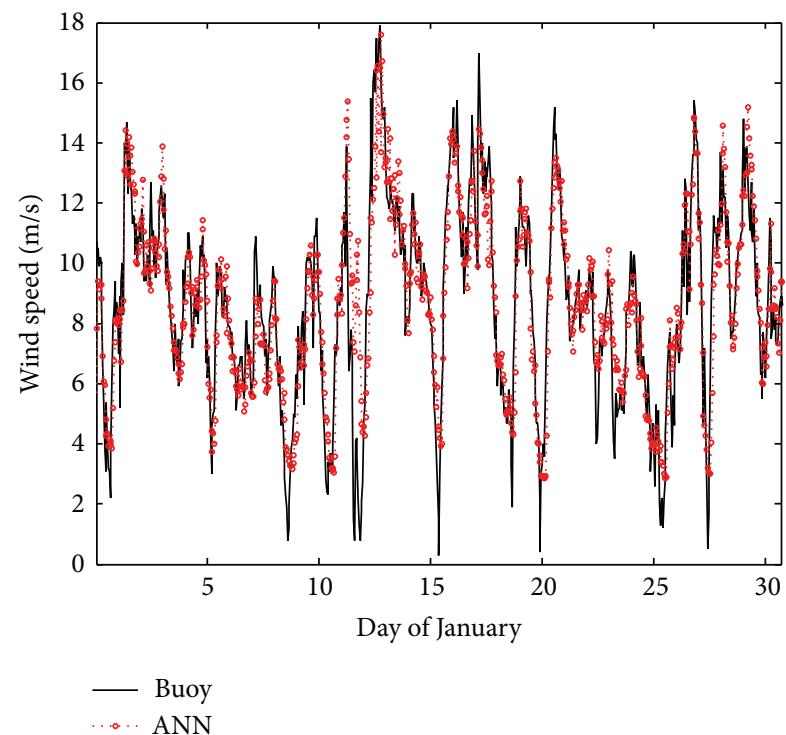

(c)

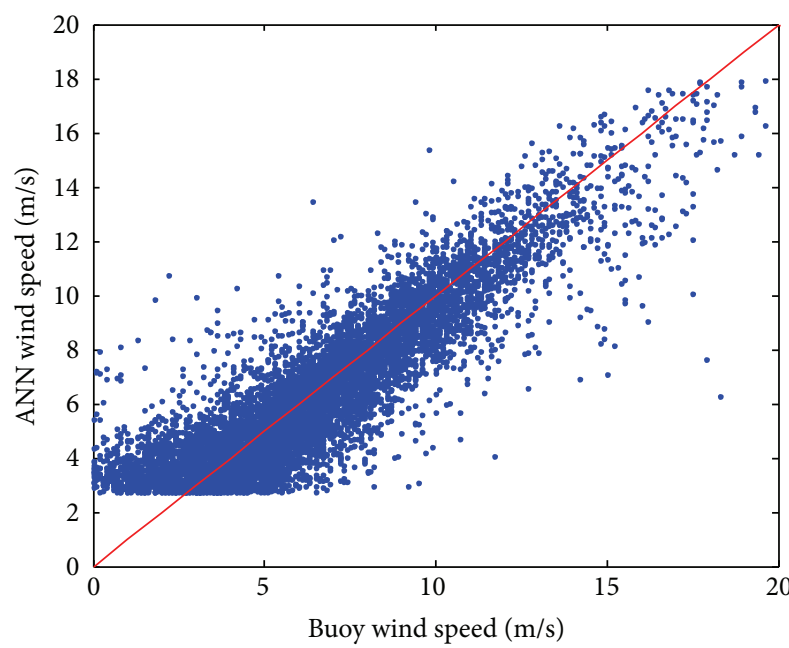

(b)

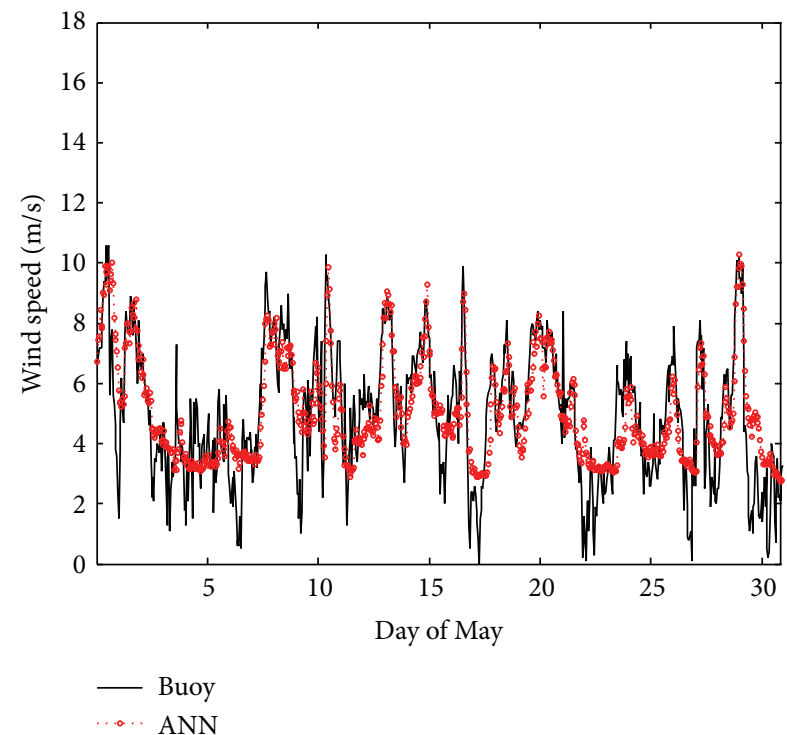

(d)

FIGURE 3: Buoy wind speed estimation in 2012 by the ANN trained with the buoy data of 2011. (a) Time sequence of the whole year. (b) Scatter plot. (c) Time sequence in January. (d) Time sequence in May.

TABLE 2: Average wave height and wind speed per month in 2011.

\begin{tabular}{|c|c|c|c|c|c|c|c|c|c|c|c|c|}
\hline & \multicolumn{12}{|c|}{ Month } \\
\hline & Jan. & Feb. & Mar. & Apr. & May & Jun. & Jul. & Aug. & Sep. & Oct. & Nov. & Dec. \\
\hline Number of pieces of data & 738 & 665 & 734 & 717 & 742 & 719 & 721 & 0 & 0 & 0 & 513 & 743 \\
\hline Wind speed $(\mathrm{m} / \mathrm{s})$ & 7.7 & 8.0 & 7.2 & 6.7 & 4.8 & 4.5 & 4.7 & - & - & - & 6.7 & 7.3 \\
\hline Wave height $(\mathrm{m})$ & 1.25 & 1.36 & 1.38 & 1.67 & 1.18 & 0.89 & 0.91 & - & - & - & 1.26 & 1.32 \\
\hline
\end{tabular}

In the training process, the learning ratio is set to be 0.05. After 141 epochs, the ANN converges. When this trained ANN is applied on the buoy data of 2012, satisfactory wind speed estimates are obtained when the wind speeds are greater than $5 \mathrm{~m} / \mathrm{s}$, as shown in Figure 3 .
For comparison, the SMB method and the power regression method are also tested with the buoy data. The results are shown in Figure 4. As can be seen, the RMSE by the ANN method varies between 1.45 and $1.80 \mathrm{~m} / \mathrm{s}$ except that it exceeds $2.2 \mathrm{~m} / \mathrm{s}$ in October and December, and the 


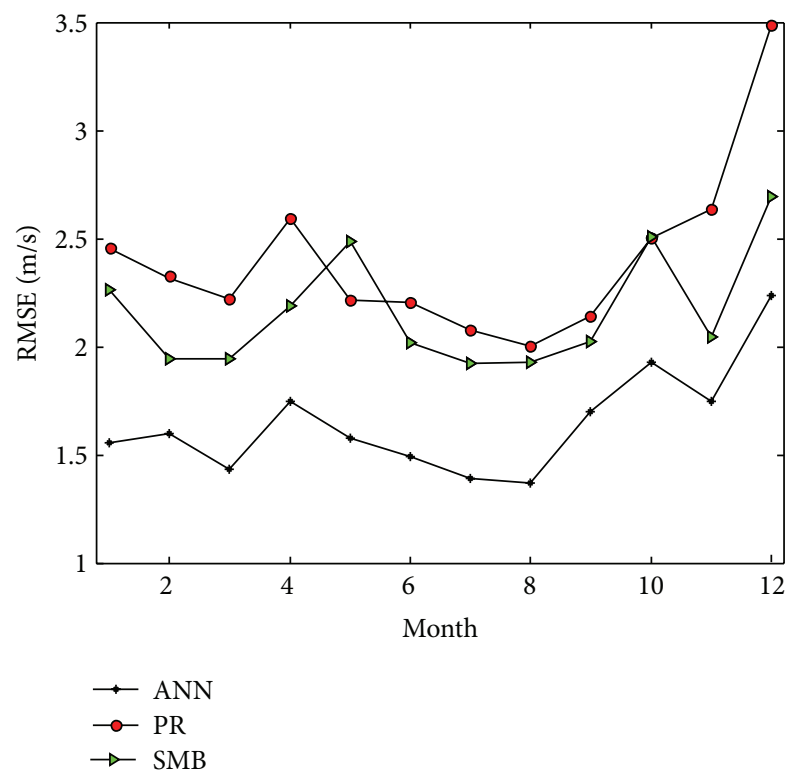

(a)

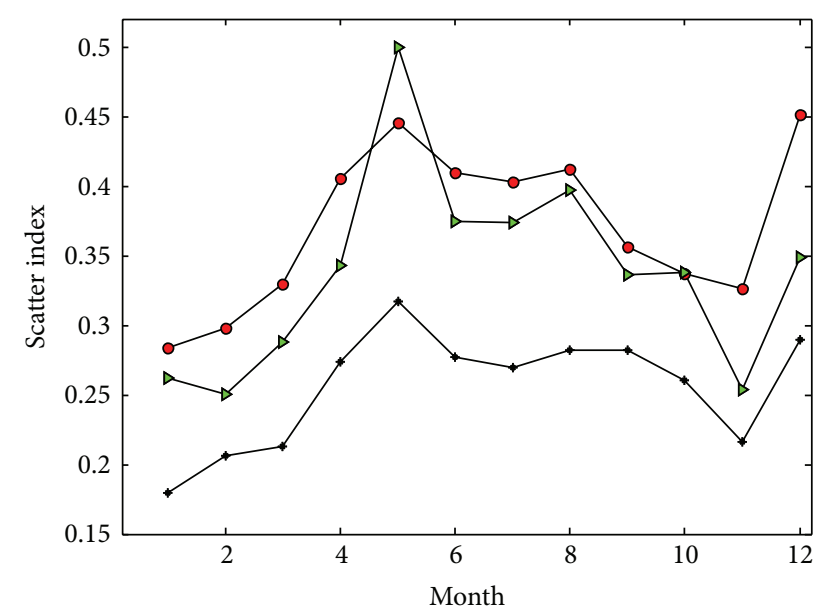

$\rightarrow$ ANN
$\rightarrow$ PR
$\rightarrow$ SMB

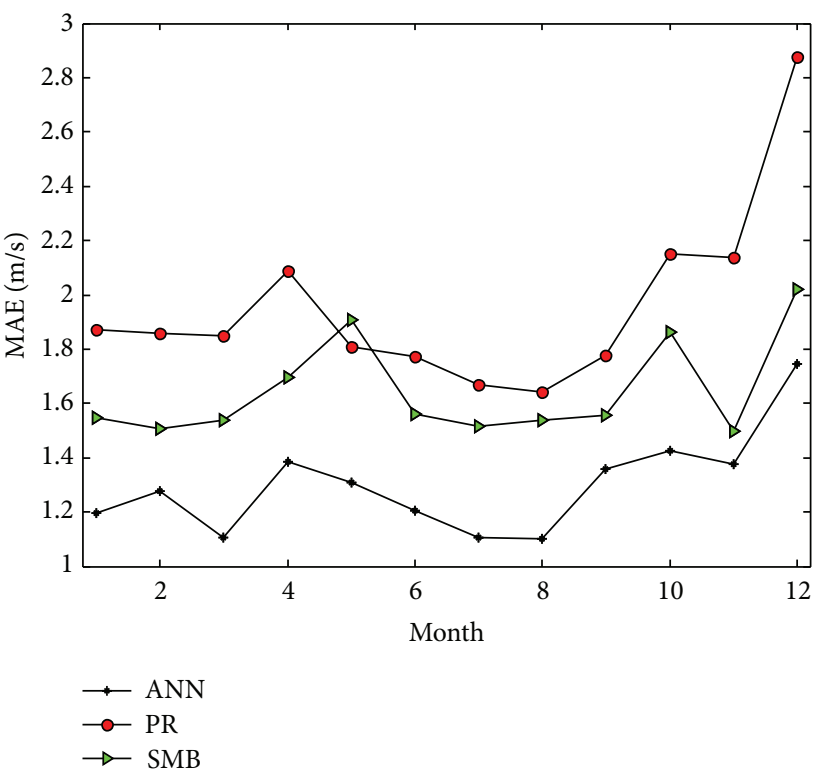

(b)
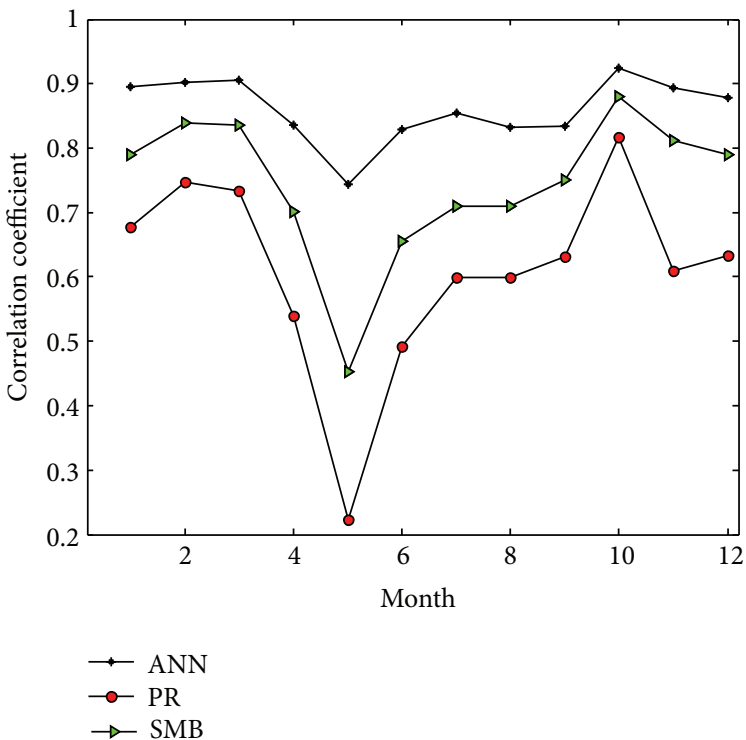

(c)

(d)

FIGURE 4: Wind speed estimated by the buoy data per month in 2012. (a) RMSE. (b) MAE. (c) CC. (d) SI.

TABLE 3: Average wave height and wind speed per month in 2012.

\begin{tabular}{|c|c|c|c|c|c|c|c|c|c|c|c|c|}
\hline & \multicolumn{12}{|c|}{ Month } \\
\hline & Jan. & Feb. & Mar. & Apr. & May & Jun. & Jul. & Aug. & Sep. & Oct. & Nov. & Dec. \\
\hline Number of pieces of data & 738 & 694 & 742 & 719 & 741 & 718 & 740 & 743 & 715 & 741 & 719 & 528 \\
\hline Wind speed (m/s) & 8.6 & 7.7 & 6.7 & 6.3 & 4.9 & 5.3 & 5.1 & 4.8 & 6 & 7.4 & 8.0 & 7.7 \\
\hline Wave height (m) & 1.54 & 1.23 & 1.25 & 1.14 & 1.14 & 0.98 & 0.91 & 0.89 & 1.22 & 1.53 & 1.53 & 1.50 \\
\hline
\end{tabular}

correlation coefficient (CC) is greater than 0.82 (up to 0.91 ) except that it is 0.78 in May. The sea state in fact has an impact on the wind speed estimation. The big errors in October and December are mainly caused by the relatively fewer training samples corresponding to the large wave heights above $5 \mathrm{~m}$, and the decrease of the correlation coefficient in May is mainly due to the low wind speed in that month. Generally, the ANN method outperforms the SMB and power regression methods in all the indices, say the RMSE, mean absolute error (MAE), correlation coefficient (CC), and scatter index (SI, 
TABLE 4: Performances of wind speed estimation from different buoys in different years by the ANN trained with the buoy data of 2008.

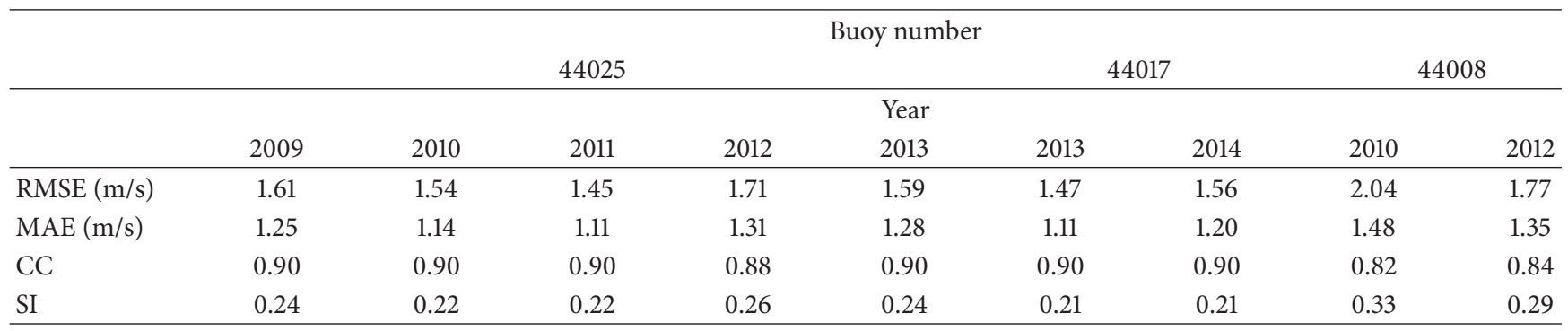

defined as the ratio of the standard error to the mean absolute value), and its estimation performance is much less sensitive to the sea states. So the ANN method is expected to offer a promising way to estimate the wind speed in HF radars.

According to Table 3, the wind speed in May is significantly lower than that in April, but the wave height is higher than that of April because of the full developed waves and swells. In terms of scatter index and RMSE, we can see that the performance of $\mathrm{PR}$ is slightly better than that of SMB. In addition to swell, it also may be the reason that the SMB method is more suitable for the wave without full development in fetch restricted conditions. In May, the results have an unsatisfactory correlation factor of about 0.45 , while they show an excellent correlation factor of 0.9 in October, as shown in Figure 4(d). These show that the performance of SMB method is not stable enough and is only suitable for the wind-derived waves in fetch restricted conditions.

Even though traditional SMB wind-wave empirical equations perform badly in swell and fully developed sea conditions, the ANN performs well in May, as Figure 4 shows. In Table 2, we can see that the wave height in April is higher than that in May, while the wind speed is smaller than that in May for swells coming to this sea area frequently in April. These training data contain the relationship between wind speed and other wave parameters in swell and fully developed sea conditions.

Then the significant wave height, wave period, and wind direction from the SeaSonde in May of 2012 are input to the trained ANN to estimate the wind speed. It should be pointed out that these input parameters are extracted not by the commercial software of the SeaSonde but by that of the OSMAR-S developed by Wuhan University, whose performance has been validated by a series of experiments [20-22]. Figure 5 shows the time sequence of the significant wave height estimates. The RMSE and CC between the significant wave heights from the radar and the buoy are $0.24 \mathrm{~m}$ and 0.79 , respectively. The wind speed estimation results are shown in Figure 6. It can be seen that the ANN method also gives better wind speed estimation than the SMB and power regression methods. The RMSE, MAE, CC, and SI of the wind speed estimation by the ANN method are $1.70 \mathrm{~m} / \mathrm{s}, 1.37 \mathrm{~m} / \mathrm{s}, 0.68$, and 0.34 , respectively.

Before it is developed into an operational method, the generalization ability of the ANN should also be considered. To evaluate the generalization ability of the ANN in both of the time and space, we further test it on the buoy data of other

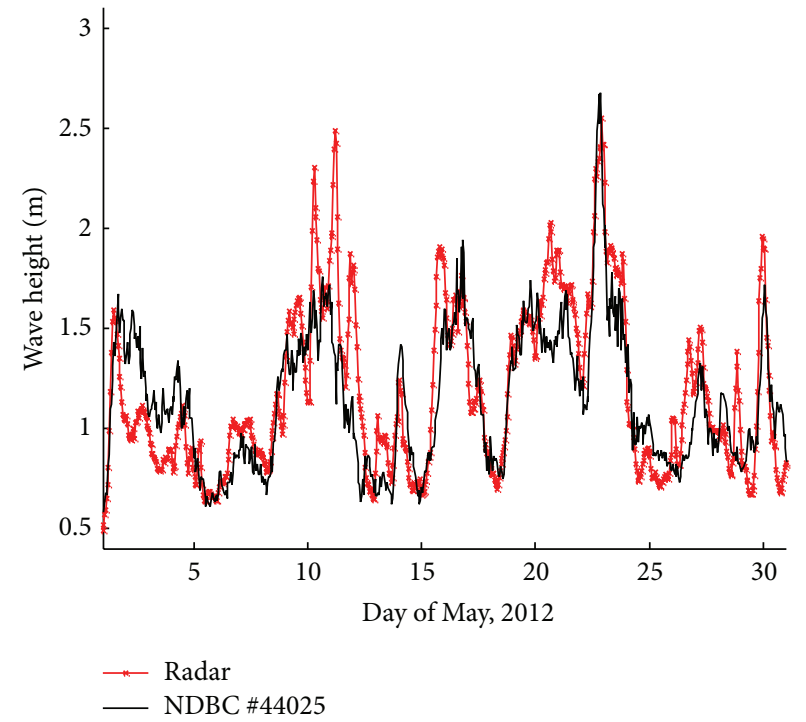

FIGURE 5: Significant wave height extracted from the SeaSonde at the BRMR site in May, 2012.

several years, say 2009 till 2013. The estimation performances in the years are listed in Table 4, from which we can see that the performance of the ANN is almost independent of the time. Even when this ANN is applied on the data of 2012, the result is still satisfactory, quite similar to that achieved by the ANN trained with the data of 2011. This shows strong generalization ability in the time. Then the ANN is applied on the data from other two NDBC buoys, say number 44017 $\left(40^{\circ} 41^{\prime} 39^{\prime \prime} \mathrm{N}, 72^{\circ} 2^{\prime} 52^{\prime \prime} \mathrm{W}\right)$ and number $44008\left(40^{\circ} 30^{\prime} 10^{\prime \prime} \mathrm{N}\right.$, $\left.69^{\circ} 14^{\prime} 53^{\prime \prime} \mathrm{W}\right)$, which are about 120 and $360 \mathrm{~km}$ away from the buoy number 44025, respectively, as shown in Figure 1. The ANN method once more shows a strong generalization ability in the space, which can be seen in Table 4 . Even when the distance exceeds $300 \mathrm{~km}$, the correlation coefficient is still above 0.8 . The result of number 44008 is slightly worse than that of number 44017, which should be because the former one is much farther from the coast and thus the bathymetry deepens faster and has a larger influence on the wind-wave relationship. Two deductions can be achieved from these results. The inherent relationship between the wind speed and wave parameter is quite stable in both time and space, and the ANN can recognize this relationship well. Since the buoys can provide in situ measurements with high accuracy while they are usually not deployed very closely to each other, 


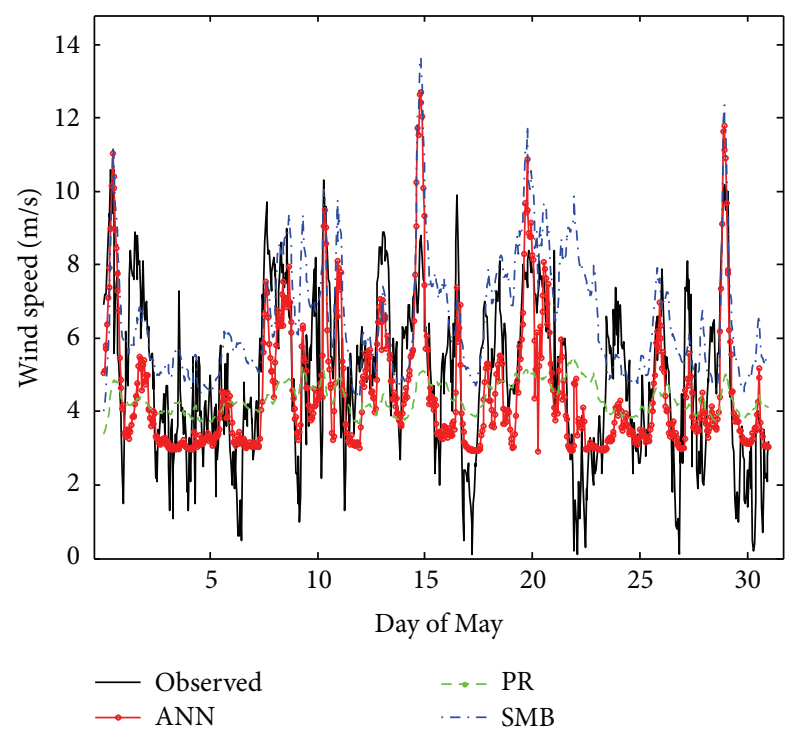

(a)

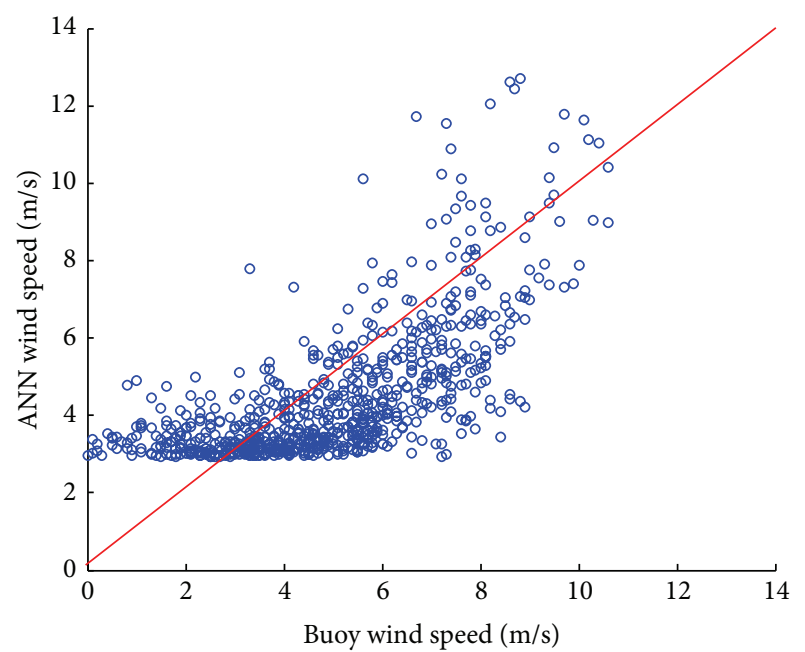

(b)

FIGURE 6: Radar wind speed estimation in May, 2012, by the ANN trained with the buoy data of 2011. (a) Wind speed estimates. (b) Scatter plot by the ANN method.

the HF radar just can accomplish the large-area monitoring task with the help of a limited number of buoys. So we can conclude that the ANN is an efficient tool to realize wind speed extraction in HF radar for operational use.

\section{Conclusions}

The artificial neural network is a powerful tool that can automatically learn and memorize the unknown, complicated relationship between some correlated parameters. The HF radar can achieve wave height, period, and wind direction estimates directly from the echo Doppler spectra, while the wind speed should be estimated in an indirect way. That is, the wind speed is estimated from the wave parameters via an empirical or semiempirical method, so the inconsistency between these models and the actual relation forms a main source of error in wind speed estimation. The wave extraction in HF radar has achieved relatively high accuracy, but the imperfect models make the wind speed estimation be still a challenge. The ANN is such an efficient tool that offers improved performance on wind speed estimation in $\mathrm{HF}$ radar. Moreover, it is available in a wide range of time and space ( 2 years around and $360 \mathrm{~km}$ away), which opens the possibility of operational use of wind speed from HF radar.

\section{Competing Interests}

The authors declare that there are no competing interests regarding the publication of this paper.

\section{Acknowledgments}

This work was supported by the National Natural Science Foundation of China under Grant no. 61371198 and the
National Special Program for Key Scientific Instrument and Equipment Development of China under Grant no. 2013 YQ160793.

\section{References}

[1] D. D. Crombie, "Doppler spectrum of sea echo at $13.56 \mathrm{Mc./s.,"}$ Nature, vol. 175, pp. 681-682, 1955.

[2] D. E. Barrick, "First-order theory and analysis of MF/HF/VHF scatter from the sea," IEEE Transactions on Antennas and Propagation, vol. 20, no. 1, pp. 2-10, 1972.

[3] D. Barrick, "Remote sensing of sea state by radar," in Proceedings of the IEEE International Conference on Engineering in the Ocean Environment (Ocean '72), pp. 186-192, Newport, RI, USA, September 1972.

[4] G. J. Koch, Y. J. Beyon, M. Petros et al., "Side-scan Doppler lidar for offshore wind energy applications," Journal of Applied Remote Sensing, vol. 6, no. 10, pp. 359-368, 2012.

[5] H. U. Sverdrup and W. H. Munk, "Empirical and theoretical relations between wind, sea, and swell," Eos, Transactions American Geophysical Union, vol. 27, no. 6, pp. 823-827, 1946.

[6] P. E. Dexter and S. Theodoridis, "Surface wind speed extraction from HF sky wave radar Doppler spectra," Radio Science, vol. 17, no. 3, pp. 643-652, 1982.

[7] W. Huang, S. Wu, E. Gill, B. Wen, and J. Hou, "HF radar wave and wind measurement over the Eastern China Sea," IEEE Transactions on Geoscience and Remote Sensing, vol. 40, no. 9, pp. 1950-1955, 2002.

[8] W. Huang, E. Gill, S. Wu, B. Wen, Z. Yang, and J. Hou, "Measuring surface wind direction by monostatic HF groundwave radar at the Eastern China Sea," IEEE Journal of Oceanic Engineering, vol. 29, no. 4, pp. 1032-1037, 2004.

[9] K. Watanabe and K. Nomura, "Investigation of ocean wind estimating technique using wave data and SMB method," in Proceedings of the 7th International Conference on Asian and Pacific Coasts (APAC '13), Bali, Indonesia, September 2013. 
[10] L. Li, X. Wu, X. Xu, and B. Liu, "An empirical model for wind speed inversion by HFSWR," Geomatics and Information Science of Wuhan University, vol. 37, no. 9, pp. 1096-1099, 2012.

[11] D. Green, E. Gill, and W. Huang, "An inversion method for extraction of wind speed from high-frequency ground-wave radar oceanic Backscatter," IEEE Transactions on Geoscience and Remote Sensing, vol. 47, no. 10, article 8, pp. 3338-3346, 2009.

[12] R. H. Stewart and J. R. Barnum, "Radio measurements of oceanic winds at long ranges: an evaluation," Radio Science, vol. 10, no. 10, pp. 853-857, 1975.

[13] T. E. Mathew and M. C. Deo, "Inverse estimation of wind from the waves measured by high-frequency radar," International Journal of Remote Sensing, vol. 33, no. 10, pp. 2985-3003, 2012.

[14] W. Shen, An algorithm to derive wind speed and direction as well as ocean wave directional spectra from HF radar backscatter measurements based on neural network [Ph.D. thesis], Universität Hamburg, 2011.

[15] S. Haykin, Neural Networks: A Comprehensive Foundation, MacMillan, New York, NY, USA, 1994.

[16] D. E. Rumelhart, G. E. Hinton, and R. J. Williams, "Learning internal representations by error propagation," in Parallel Distributed Processing: Explorations in the Microstructure of Cognition, vol. 1, pp. 318-362, MIT Press, Boston, Mass, USA, 1986.

[17] F. D. Foresee and M. T. Hagan, "Gauss-Newton approximation to Bayesian learning," in Proceedings of the IEEE International Conference on Neural Networks, vol. 3, pp. 1930-1935, IEEE, Houston, Tex, USA, June 1997.

[18] D. J. MacKay, "A practical Bayesian framework for backpropagation networks," Neural Computation, vol. 4, no. 3, pp. 448-472, 1992.

[19] H. Roarty, C. Evans, S. Glenn, and H. Zhou, "Evaluation of algorithms for wave height measurements with high frequency radar," in Proceedings of the IEEE/OES 11th Current, Waves and Turbulence Measurement (CWTM '15), pp. 1-4, March 2015.

[20] B.-Y. Wen, Z.-L. Li, H. Zhou et al., "Sea surface currents detection at the eastern china sea by HF ground wave radar OSMAR-S," Acta Electronica Sinica, vol. 37, no. 12, pp. 27782782, 2009.

[21] H. Zhou, H. Roarty, and B. Wen, "Wave extraction with portable high-frequency surface wave radar OSMAR-S," Journal of Ocean University of China, vol. 13, no. 6, pp. 957-963, 2014.

[22] H. Zhou, H. Roarty, and B. Wen, "Wave height measurement in the Taiwan Strait with a portable high frequency surface wave radar," Acta Oceanologica Sinica, vol. 34, no. 1, pp. 73-78, 2015. 


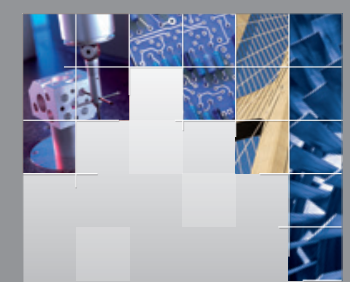

\section{Enfincering}
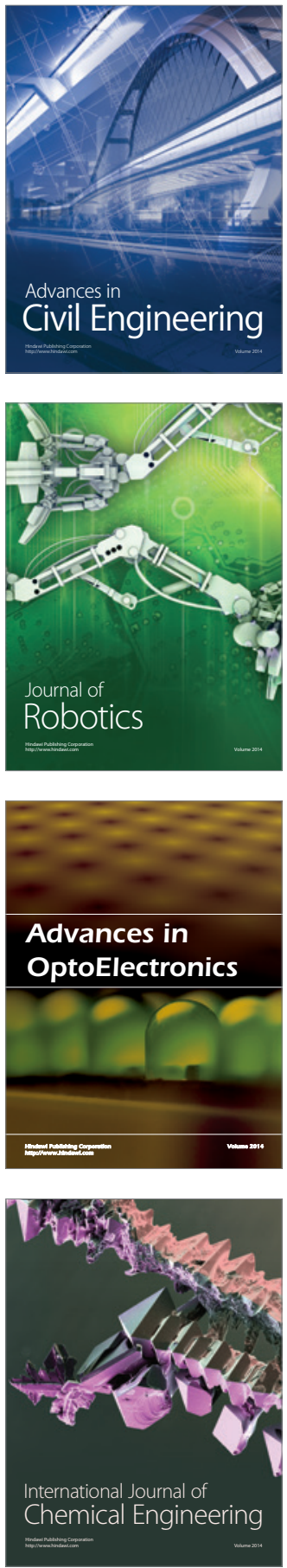

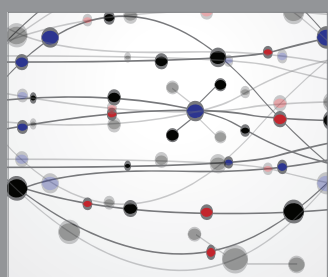

The Scientific World Journal

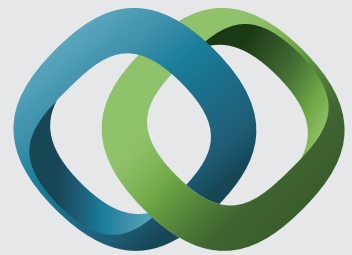

\section{Hindawi}

Submit your manuscripts at

http://www.hindawi.com
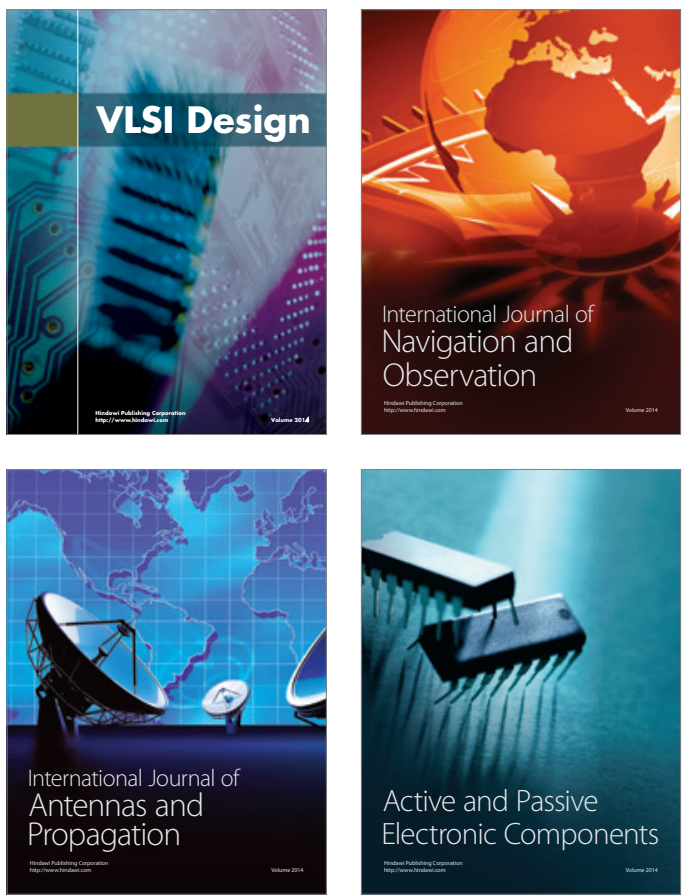
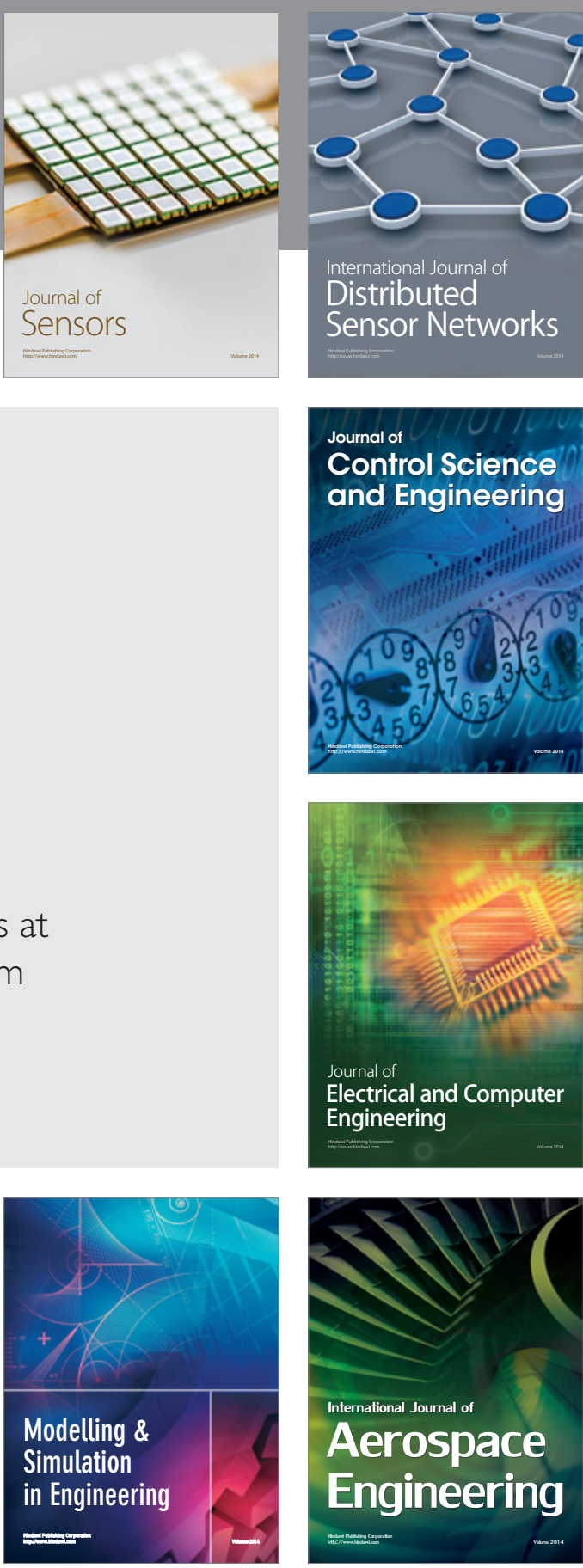

International Journal of

Distributed

Sensor Networks

Journal of

Control Science

and Engineering
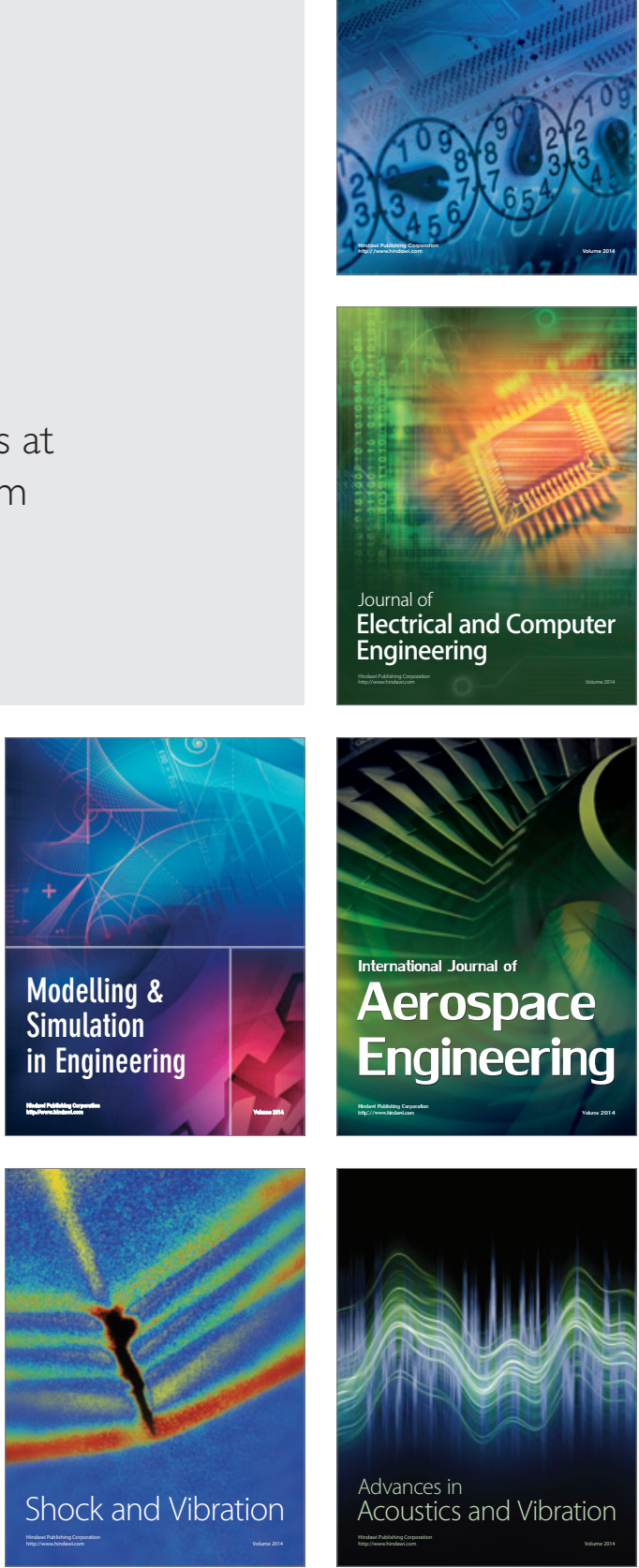